\section{Limits of Application for Two-Dimensional Elasticity Solutions in Plates Loaded in Tension and Out-of-Plane Bending*}

\author{
Yo-ichiro KITAHARA**, Hiroshi NOGUCHI** and Toyomitsu HARADA*** \\ **Faculty of Engineering, Kyushu University, \\ 744 Motooka, Nishi-ku, Fukuoka 819-0395, Japan \\ ${ }^{* * *}$ Kurume National College of Technology, \\ 1-1-1 Komorino, Kurume, Fukuoka 830-8555, Japan \\ E-mail: harada@kurume-nct.ac.jp
}

\begin{abstract}
Elasticity solutions of plates have been widely researched and used in practice. However, elasticity solutions of plane problems are applicable to structures only under the premise that such structures are very thin. The precise values of thickness for which elasticity solutions are valid have not been studied in detail. It is necessary to clarify the range of appropriate thickness values in order to apply two-dimensional elasticity solutions properly. In this study, we use finite element analyses to study three flat-plate models each containing a U-shaped hole of the same radius, and compared the three-dimensional finite element solutions with two-dimensional elasticity solutions. From the results, the limits of application of two-dimensional elasticity solutions are discussed.
\end{abstract}

Key words: Elasticity, Notch, Stress Concentration, Plane Problem, Two-Dimensional Elastic Solution, Three-Dimensional Stress Analysis, Limits of Application, FEM

\section{Introduction}

In most cases, fatigue fractures begin in a stress concentration region. Therefore, it is important to consider stress concentration factors in engineering analyses. Finite element analyses can be conducted to calculate precise values of stress concentration factors. However, it is often necessary to obtain an approximate value or to evaluate quickly whether the results from a numerical analysis are appropriate or not. A two-dimensional elastic solution is often used in such cases for the following reasons;

1. Accurate values are described in handbooks ${ }^{(1)}$.

2. Some of these solutions are described as a positive type, so they are easy to understand $^{(2) \sim(4)}$. The solution for an elliptical hole in an infinite plate is especially important.

3. Two-dimensional elastic solutions are widely available by using positive-type analytic solutions and the equivalent ellipse concept ${ }^{(5),(6)}$ together.

Two-dimensional stress conditions, namely the plane stress condition and the plane strain condition, are mathematically defined as conditions of an infinitely thin plate and that

\footnotetext{
*Received 13 Sep., 2010 (No. T2-07-0896) Japanese Original : Trans. Jpn. Soc. Mech. Eng., Vol. 74, No. 741, A (2008), pp.647-654 (Received 9 Oct., 2007) [DOI: 10.1299/jmmp.4.1778]
}

Copyright () 2010 by JSME 
of inside of an infinitely thick plate, respectively. From these idealized definitions, it is necessary to clarify the limits of application in practice for engineering analyses for two-dimensional elastic solutions. We compare two-dimensional stress fields of various shapes with their three-dimensional stress fields to use two-dimensional elastic solutions effectively.

In three-dimensional stress fields, the stress concentration factor in a finite thick plate having a circular hole under uniform tension has been studied by varying Poisson's ratio and the ratio of the circle radius to the plate thickness ${ }^{(7)}$. The stress concentration factor of an infinite plate having a circular hole under out-of-plane deformation was studied by varying the ratio of the circle radius to the plate thickness ${ }^{(8)}$. However, these studies considered only a circular hole. Thus their results cannot be used for holes of various shapes.

In the present study, the authors conduct two-dimensional and three-dimensional elastic analyses of plates having a U-shaped hole using finite element method. U-shaped holes are more general than circular holes, because their notch depth can be changed. The loads that are applied are either tension loads or out-of-plane bending loads. The ratio of the hole width to the notch-root radius as well as Poisson's ratio are both variable. We present the limits of application in practice of two-dimensional elastic solutions for holes of arbitrary shape by normalizing the results of our analyses.

\section{Nomenclature}

$B$ : thickness of a plate

$M$ : bending or torsional moment

$P$ : tensile load

$t$ : notch depth

$W$ : half of width of a plate

$x$ : horizontal coordinate originated at a notch root of a surface

$y$ : vertical coordinate

$z$ : coordinate of thickness direction

$\alpha$ : stress concentration factor

v. Poisson's ratio

$\rho$ : notch-root radius

\section{Subscripts}

$e$ : equivalent elliptical hole

max: maximum

$o$ : nominal stress

II : two-dimensional

III: three-dimensional

\section{Analytical Model}

An analytical model of the present study is shown in Figure 1. It is a plate that has a central U-shaped hole. Here $\rho, 2 t$, and $B$ are the radius of curvature at the hole root, the width of the hole, and the plate thickness,

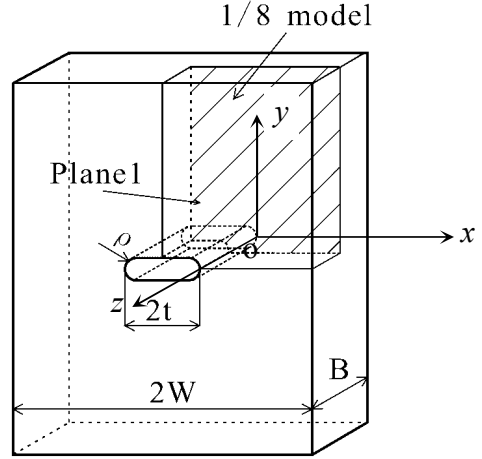

Fig.1 Analytical model 
respectively. (We call $\rho$ as "notch-root radius" and $t$ as "notch depth" hereafter, because the stress field of a hole is similar to that of a notch, and the terms "notch-root radius" and "notch depth" are usually used).

The ratio $B / \rho$ is varied from 0.2 to 2.1. Fig. 2 shows a plane shape of the model in Fig. 1 (quarter shape). The notch-root radius is 0.5 and the ratio $t / \rho$ is set at $0.4,1.0$, and 2.0. The length of each plate is made twice as large as the plate width, considering the Saint-Venant's principle, in order to realize a load condition equal to a load at infinity.

In two-dimensional analyses, 8-node plane stress and plane strain elements are used for tension and 8-node thin shell elements and 8-node thick shell elements are used for out-of-plane bending. A symmetry condition is set on planes 2 and 3 in Fig. 2. A symmetry condition is set on planes 2 and 3 in Fig. 2. A uniform tension load, $P$, or out-of-plane bending moment, $M$, is applied on the upper-end face. For the three-dimensional analyses, 20-node three-dimensional solid elements are used. A symmetry condition is given on plane 1 (Fig.1) and planes 2 and 3 (Fig.2). A uniform tension load, $P$, is applied on the upper-end face in tension. For out-of-plane bending, an antisymmetry condition on plane 1 and a symmetry condition on planes 2 and 3 are given. A uniform out-of-plane bending moment, $M$, is applied on the upper-end face.

The width of elements around the notch root is about $1 / 20$ of $\rho$ in both two- and three-dimensional analyses. The mesh division near the notch root is shown in Fig.3. Young's modulus, E, is set at $206 \mathrm{GPa}$, and Poisson's ratio, $v$, is set to be $0,0.3$, and 0.5 . (The exact value is 0.49 . For simplicity, we use the nominal value 0.5 in the following discussion.)

The stress concentration factor, $\alpha$, is defined by the following equation:

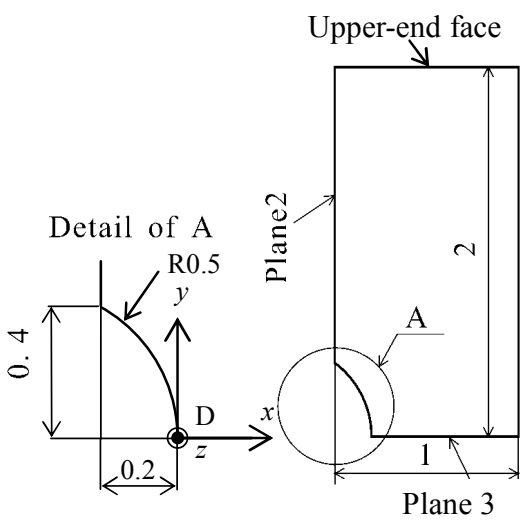

(a) $t / \rho=0.4$

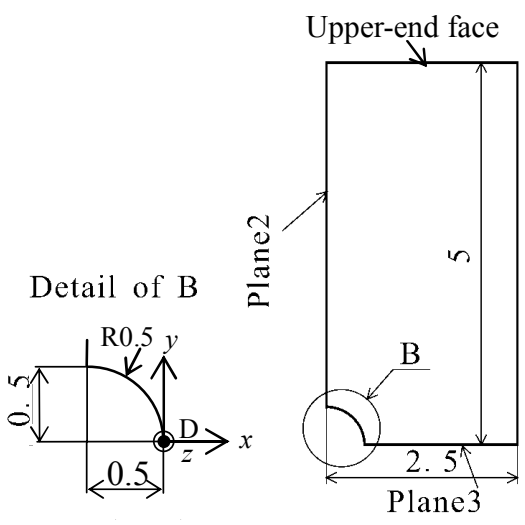

(b) $t / \rho=1$

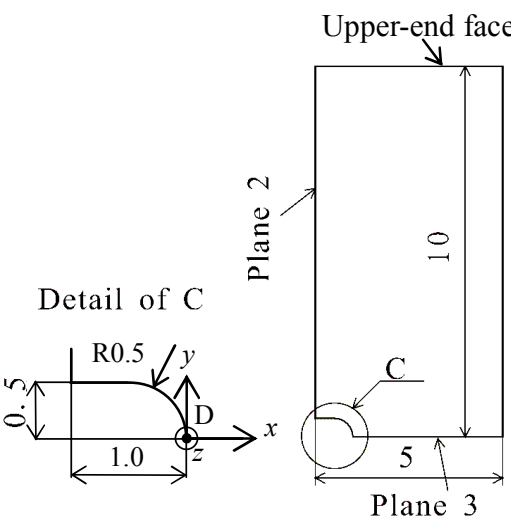

(c) $t / \rho=2$

Fig.2 Models of FEM (quarter shape)

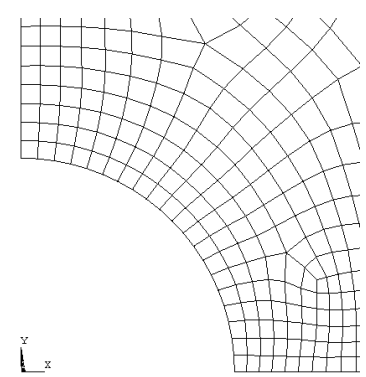

Fig.3 Mesh division near a notch root 


$$
\alpha=\frac{\sigma_{\max }}{\sigma_{o}}
$$

Here $\sigma_{\max }$ is the maximum stress at the notch root (point D in Fig.2), which is the maximum stress on a face in out-of-plane bending. The term $\sigma_{o}$ is the mean stress at the minimum cross section as defined by Eqs. (2) and (3).

In tension,

$$
\sigma_{o}=\frac{P}{2(W-t) B}
$$

In out-of-plane bending,

$$
\sigma_{o}=\frac{6 M}{2(W-t) B^{2}}
$$

The maximum stress, $\sigma_{\max }$, varies along the thickness direction, even in tension, in three-dimensional analyses. The stress concentration factor in the three-dimensional analyses, $\alpha_{\mathrm{III}}$, is defined as a function of the coordinate of thickness direction, $z$ :

$$
\alpha_{\text {III }}(z)=\frac{\sigma_{\max }(z)}{\sigma_{0}}
$$

where, $\alpha_{\mathrm{II}}$ denotes a two-dimensional stress concentration factor.

\section{Result of Numerical Analysis}

\subsection{Qualitative Prediction of the Three-Dimensional Stress Field under Tension}

The distributions of $\sigma_{y}$ in the models in Fig. 2 (a)-(c) are shown in Fig. 4. The tension loads are adjusted so that the maximum stresses at the notch root are equal. The origin of the coordinate $x$ is taken at the notch root, and the coordinate is made dimensionless by dividing it by $\rho$. The stress distributions are almost the same regardless of the ratio $t / \rho$. Thus, the two-dimensional stress field near the notch root is invariant. The stress field is expressed by a single master curve, which is made dimensionless by dividing the stress of the load direction, $\sigma_{y}$, by the maximum stress at the notch root, $\sigma_{\max }$, and dividing the coordinate, $x$, by the notch-root radius, $\rho$. Namely, the intensity of the stress field can be expressed by a stress concentration factor. The shape of the stress distribution is determined by the notch-root radius. Other factors describing

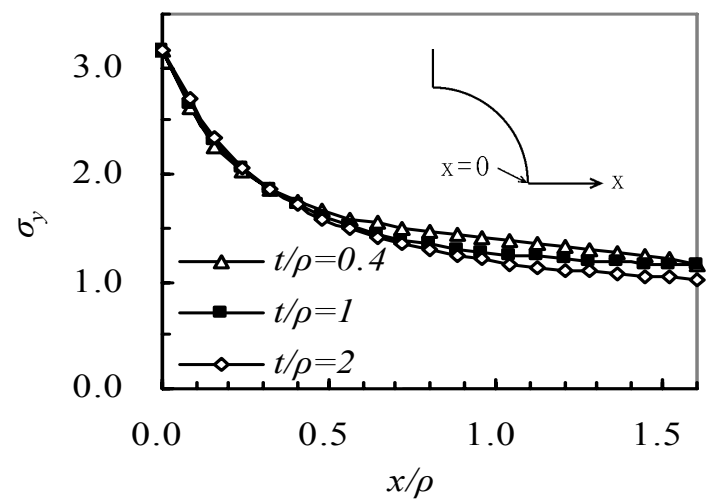

Fig.4 Distributions of $\sigma_{y}$ in two-dimensional models under uniform tension 
the notch shape, such as the notch depth, $t$, hardly influence the stress field near the notch root (which is a known result in linear notch mechanics ${ }^{(9)}$ ).

An appropriate three-dimensional stress field of a plate of finite thickness under tension loading can be obtained by releasing $\sigma_{z}$ of the plane strain problem on a plate surface. Here, $z$ is the coordinate of the thickness direction. The above-mentioned $\sigma_{z}$ is generated by restraining the deformation in the $z$-direction. The cause of the deformation in the $z$-direction is the Poisson's effect of deformation on the $x-y$ plane. According to linear notch mechanics, the approximate values of $\sigma_{z} / \sigma_{z, \max }$ $\left(\sigma_{z, \text { max }}: \sigma_{z}\right.$ at a notch root) near a notch root on a plate surface depend on $\rho$ and $v$, regardless of $t$. The $\sigma_{z} / \sigma_{z, \max }$ distributions near the notch root of the two-dimensional models shown in Fig. 2 (a)-(c) are shown in Fig. 5. The load is a uniform tension load, and the plane strain condition is assumed. Figure 5 shows that varying the ratio $t / \rho$ affects the values of $\sigma_{z} / \sigma_{z, \max }$ a little, however, the distributions of $\sigma_{z} / \sigma_{z, \max }$ for equal values of $v$ are quite similar.

From the above mentioned, the notch-root radius is an important parameter in determining three-dimensional stress fields near a notch root. Therefore, in our present study, coordinates for stress distributions are made dimensionless by dividing by the notch-root radius. The notch depth, $t$, and plate thickness, $B$, are also made dimensionless by dividing by the notch-root radius.

Although the release of $\sigma_{z}$ on the surface of a plate has pronounced effects near the

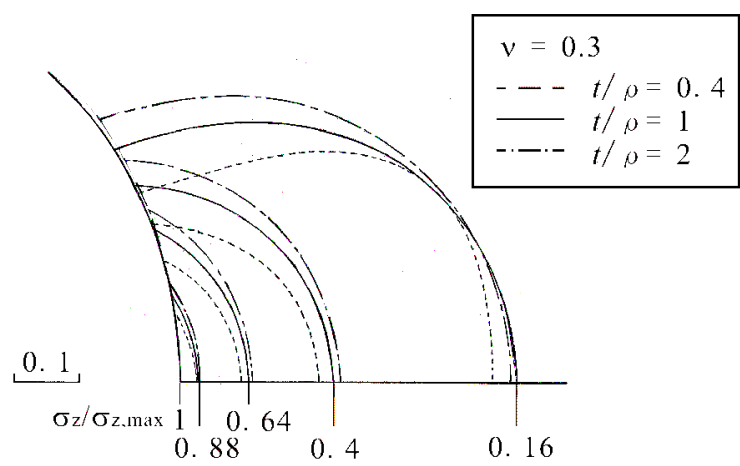

(a) $v=0.3$

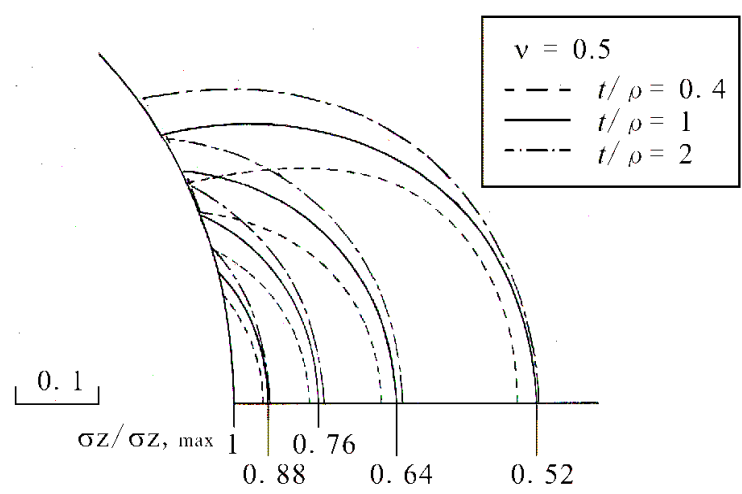

(b) $v=0.5$

Fig.5 Distribution of $\sigma_{z}$ near the notch root under the plane strain condition 
surface, its effects are not seen far inside of a thick plate. However, in a thin plate, its effects affect the whole plate. This means that the plate thickness, $B$, affects the three-dimensional stress field near a notch from the surface to the inside of the plate.

Consequently, it is predicted that the three-dimensional stress fields near a notch of a finitely thick plate are affected by $\sigma_{\max }, \rho, B$, and $v$. The influence of $t$ on the stress fields is expected to be small.

\subsection{Tension}

The distributions of the maximum stress at the notch root along the thickness direction of the models in Fig. 2(a)-(c) under uniform tension are shown in Fig. 6 . The ratio $B / \rho$ is set at $0.4,1.0$ and 2.1. $\sigma_{\max I I}$ is the maximum stress at the notch root obtained using two-dimensional stress analysis. The origin of the $z$-direction is located on a surface of the plate. The figure shows that for equal values of $B / \rho$ and $v$, the ratio of $\sigma_{\max } / \sigma_{\max I I}$ is nearly constant near the surface of the plate (i.e., when $z / \rho \leq 0.2$ ). The dimensionless notch depth, $t / \rho$, hardly affects the stress field near the surface of the plate. The stress field is determined almost exclusively by the values of $\rho, B$, and $v$. A plateau appears in the stress distribution inside the plates when $t / \rho=0.4$ and $B / \rho=2.1$ for Poisson's ratio, $v=0.3$ and 0.5 . This indicates that the inside of the plate is in the plane strain condition in these cases. When Poisson's ratio is set to 0.5 , the stress distributions are flat near the central plane for $t / \rho=1.0$ or 2.0 of $B / \rho=1.0$, too. Note that a plane strain

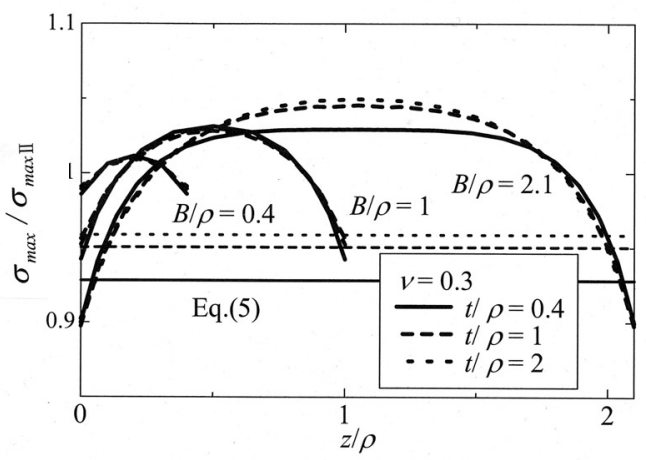

(a) $v=0.3$

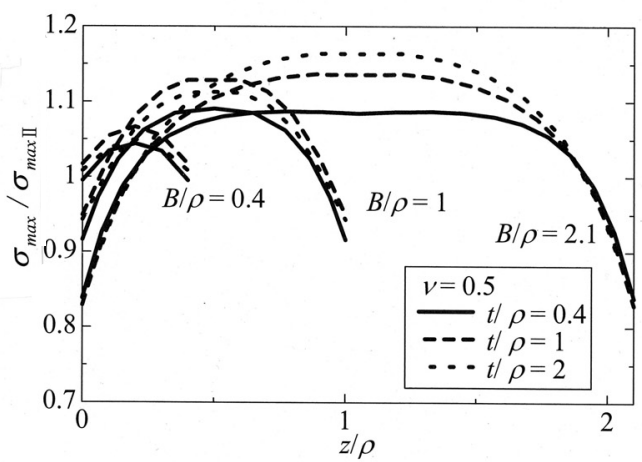

(b) $v=0.5$

Fig.6 Distribution of $\sigma_{\max }$ along the thickness direction $\left(\sigma_{\max I I}\right.$ : Maximum stress in two-dimensional) 
condition is realized in thinner plates in cases where Poisson's ratio, $v=0.5$, as compared with cases where $v=0.3$.

The stress near the surface decreases and that in the interior of the plate increases as a consequence of larger values of $B / \rho$ and $v$. The cause of these effects is as follows. Adding $-\sigma_{z}\left(\sigma_{z}\right.$ : the normal stress in $z$-direction of the plane strain condition) to the stress field of the plane strain condition generates compressive $\sigma_{y}$ near the root of the notch because of Poisson's effect. The compressive $\sigma_{y}$ is large near the surface in the neighborhood of the notch root, where $\sigma_{y}$ decreases. When the decrease of $\sigma_{y}$ near the surface is large, $\sigma_{y}$ increases inside the plate because of equilibrium of $\sigma_{y}$ and the outside load.

When $B / \rho$ and $v$ are both large, the difference in stress distributions due to the effect of value of $t / \rho$ is also large. This effect occurs for two reasons. First, the stress distributions of $\sigma_{z}$ in a region distant from the notch root under the plane strain condition differs for different values of $t / \rho$ as shown in Fig. 5. Second, Poisson's effect of releasing the stress $\sigma_{z}$ on the surface far from the notch root appears even near a central plane in thick plates.

Given the above facts, in the cases where $B / \rho$ and $v$ are large, the difference between $\sigma_{\max }(z)$ and $\sigma_{\max I}$ is also large. In particular, in the center of a plate, $\sigma_{\max }(z)$ is larger than $\sigma_{\max I I}$ by a maximum of $15 \%$. Two-dimensional elasticity solutions should be applied carefully to thick plates made of materials of large Poisson's ratio, because static fracture can occur due to large stress at the center of the plate thickness. A stress concentration factor is approximated from the following equation from the equivalent ellipse concept ${ }^{(5),(6)}$

$$
\alpha_{e}=\left(1+2 \sqrt{\frac{t}{\rho}}\right) \frac{W-t}{W}
$$

The ratios of stress concentration factors estimated using Eq. (5) divided by those obtained using a two-dimensional analysis are shown by straight lines in Fig. 6(a). The data in the figure shows that the two-dimensional stress concentration factors found in the present study are approximated with an error of less than $10 \%$ by the equivalent ellipse concept. Furthermore, errors in the stress distributions calculated using Eq. (5) (again shown by straight lines of stress concentration factors) for stress distributions calculated using a three dimensional analysis are also less than $10 \%$ over the whole plate thickness, when $v=0.3$. This data indicate that the equivalent ellipse concept is effective for approximating three-dimensional stress concentration factors.

The relationship between the ratio of stress concentration factors of three-dimensions to those of two-dimensions, $\alpha_{\mathrm{III}} / \alpha_{\mathrm{II}}$, and the ratio $B / \rho$ on the surface of a plate is shown in Fig. 7(a). Similar results calculated at the central plane of the plate are shown in Fig. 7(b). Poisson's ratios of 0.3 and 0.5 are both used. When $B / \rho=0$, the model is two-dimensional. The figures indicate that the effect of the ratio $t / \rho$ on the difference between stress concentration factors obtained using three- and two-dimensional analyses is largest in the central region of the plate. From the analyses of Figs. 6 and 7 , three-dimensional stress fields are determined by $\rho$, the ratio $B / \rho$, and $v$ near a surface of the plate and are also affected by the ratio $t / \rho$ near the center of plate thickness. The effect is larger when the ratio $B / \rho$, and $v$ are larger. 


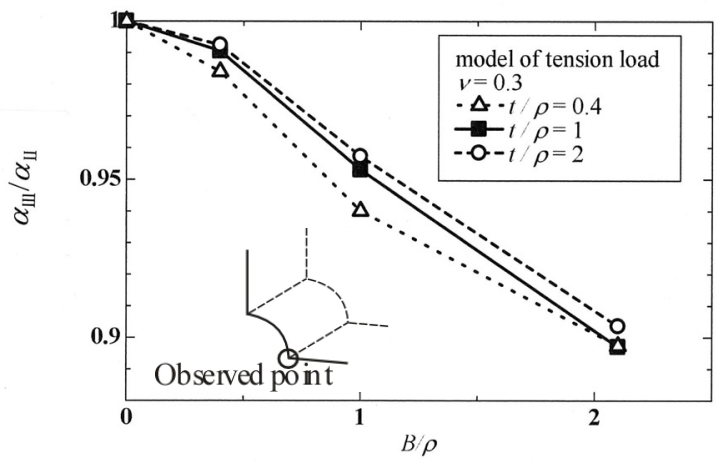

(a) $v=0.3$

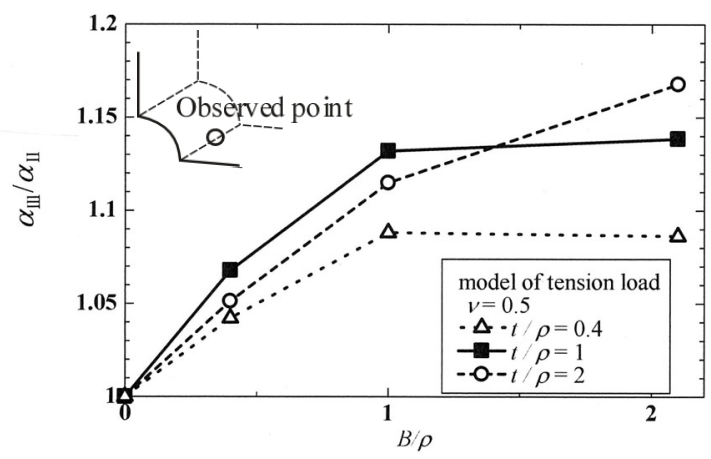

(b) $v=0.5$

Fig.7 Relationship between the ratio of three-dimensional and two-dimensional stress concentration factors, $\alpha_{\mathrm{II}} / \alpha_{\mathrm{II}}$ and $B / \rho$, under uniform tension

\subsection{Out-of-plane Bending}

The distribution of $\sigma_{y}$ at the notch root along the plate-thickness direction under uniform out-of-plane bending is shown in Fig. 8. The models are shown in Fig. 2(a)-(c). The ratio $B / \rho$ is set at $0.2,0.4$, and 1.0. The mean stress at the surface of the minimum cross section, $\sigma_{o}$, is shown in Eq. (3). The stress distributions when $B / \rho=0.1$ as obtained using the equivalent ellipse concept are also shown. They are assumed to be straight distributions.

We obtain a stress concentration factor under a uniform out-of-plane bending moment from the exact solution of an infinite thin plate ${ }^{(10)}$ as follows:

$$
\alpha=\left\{1+\frac{2(1+v)}{3+v} \sqrt{\frac{t}{\rho}}\right\} \frac{W-t}{W}
$$

From Fig. 8, the stress distributions change from straight lines to being curved as the plate thickness increases.

Figure 9 shows the relationship between the ratio of the three-dimensional stress concentration factor to the two-dimensional stress concentration factor, $\alpha_{\text {III }} / \alpha_{\text {III }}$, for varying values of $B / \rho$. The models are shown in Fig. 2(a)-(c). It is obvious that the difference between the two- and three-dimensional stress concentration factors is larger as the ratio $B / \rho$ increases and $t / \rho$ decreases. However, this difference due to $t / \rho$ when $B / \rho=1.0$ is only about $7 \%$. Therefore, the notch depth, $t / \rho$, does not have a large affect 


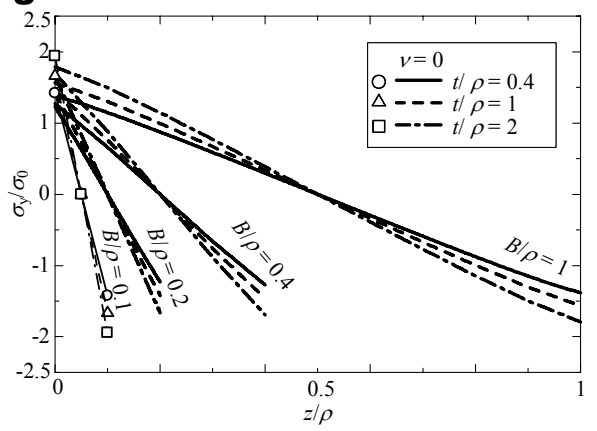

(a) $v=0$

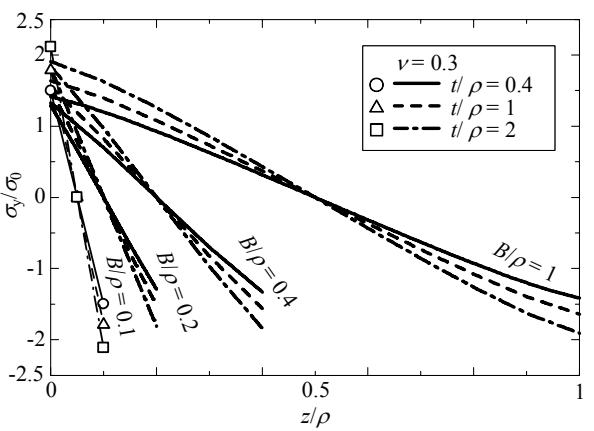

(b) $v=0.3$

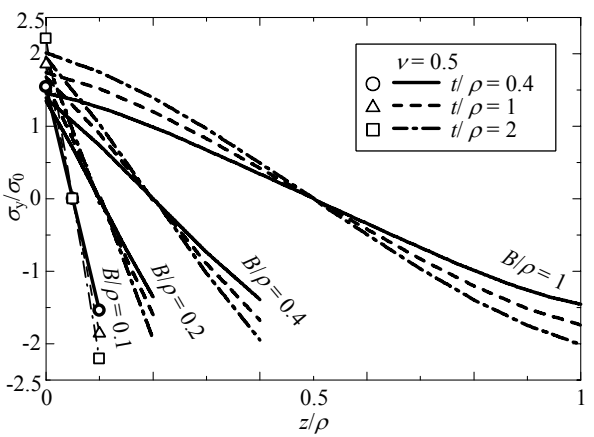

(c) $v=0.5$

Fig.8 Distribution of $\sigma_{y} / \sigma_{0}$ at the notch root along the thickness direction under a bending moment

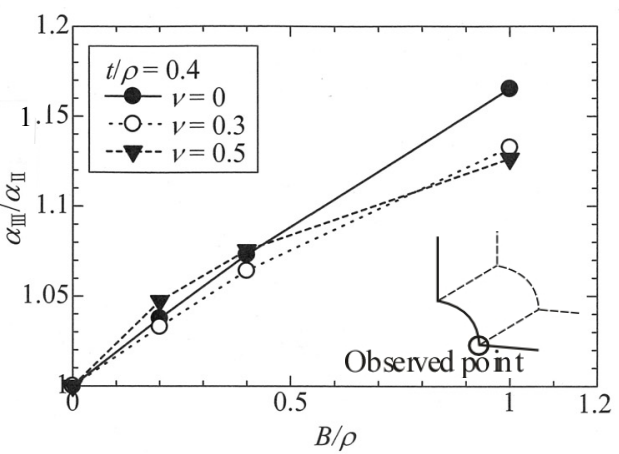

(a) $t / \rho=0.4$

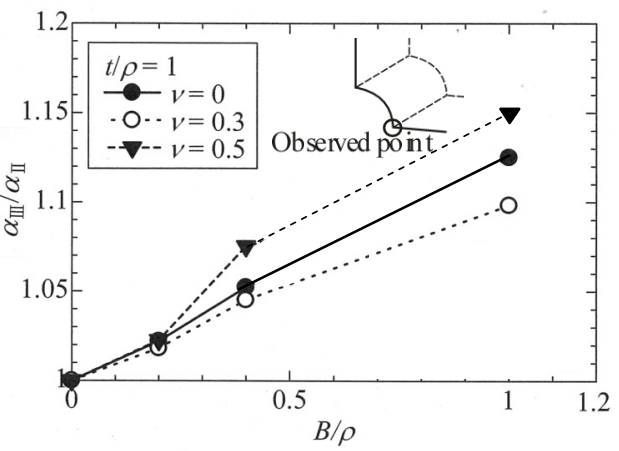

(b) $t / \rho=1.0$

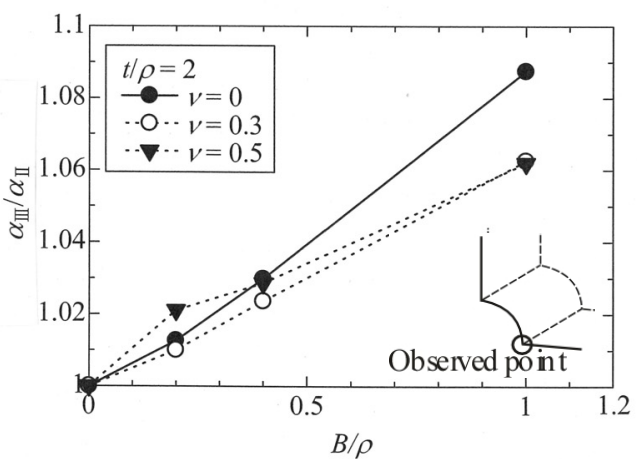

(c) $t / \rho=2.0$

Fig. 9 Relationship between $\alpha_{\text {III }} / \alpha_{\text {II }}$ and $B / \rho$ under a bending moment

on the difference between the stress concentration factors in two-dimensions and three-dimensions. The difference between the stress concentration factors in two-dimensions and three-dimensions due to Poisson's ratio, when $t / \rho$ is maintained constant, is only about $4 \%$ maximum. The effect of Poisson's ratio itself is small. The difference between the stress concentration factor in two-dimensions and three-dimensions does not exceed $10 \%$ when $B / \rho \leq 0.5$ in the present analyses. The difference between the stress concentration factors on the surface of a two- and a three-dimensional model tends to be larger in out-of-plane bending than in tension, as seen in Figs. 7 and 9. For example, in Fig. $7(\mathrm{~b}), B / \rho$ is 0.7 when the difference between the stress concentration factor in two-dimensions and three-dimensions reaches $10 \%$. But under the conditions shown in Fig. 9(a), the value of $B / \rho$ is only 0.57 .

To investigate this further, the cases where the ratios $B / \rho$ and $t / \rho$ both equal 1.0 are analyzed using thick shell elements. The Poisson's ratio, $v$, is set at 0 and 0.5. The stress concentration factors are shown in Table 1 for analyses using thin shell elements, 
thick shell elements and three-dimensional solid elements. Furthermore, exact values calculated by E. Reissner when $v=1 / 3$ are shown in the lowest line. The value in parentheses is obtained by dividing the stress concentration factors obtained using thick shell elements and three-dimensional solid elements by those obtained using thin shell elements (when $v=1 / 3$, as is the case in the classical theory of bending thin plates). From the table, although the stress concentration factors obtained using thick shell elements are larger than those obtained using three-dimensional solid elements, they are closer to the values obtained using three-dimensional solid elements than those obtained using thin shell elements. The difference between the stress concentration factors obtained using thick shell elements and those obtained using three-dimensional solid elements is between $2 \%$ and $4 \%$. This is approximately the same as the difference between stress concentration factors in two-dimensions and three-dimensions with tension loading, where $B / \rho=1.0$, $t / \rho=1.0$, and $v=0.3$, as shown in Fig. 7(a). The following explanations are possible causes of this phenomenon.

For thick shell elements, based on the theory by E. Reissner ${ }^{(8)}$, although the distributions of $\sigma_{x}, \sigma_{y}$, and $\tau_{x y}$ the along thickness direction are all linear, the bending moments $M_{x}, M_{y}$, and torsional moment $M_{x y}$ can all be set as boundary conditions. Furthermore, in elements experiencing plane stress and plane strain, although $\sigma_{x}, \sigma_{y}$, and $\tau_{x y}$ are constant along the thickness direction, they can also be set as boundary conditions. Thus, a thick shell element is the same as a plane stress element and a plane strain element at the viewpoint that the loads corresponding to $\sigma_{x}, \sigma_{y}$, and $\tau_{x y}$ can be given as the boundary conditions, under the limitation of stress distribution along the thickness direction. Therefore, the difference between the stress concentration factors obtained using thick shell elements and those obtained using three-dimensional solid elements is the same degree as the difference between the stress concentration factors obtained using the plane elements and those obtained using three-dimensional solid element.

For thin shell elements, based on the classical theory of bending of thin plates ${ }^{(1)}$, for simplicity it is assumed that the plane vertical to the neutral plane is still vertical to it after deformation. For that case, an equivalent shearing force ${ }^{(12)}$ is introduced using an out-of-plane shearing force and a torsional moment (noting that a torsional moment is never used alone.) Consequently, the torsional moment, $M_{x y}$, corresponding to $\tau_{x y}$ cannot be given as a boundary condition. Hence the difference between the stress concentration factors obtained using thin shell elements and those obtained using three-dimensional solid elements is larger than the difference between stress concentration factors obtained using thick shell elements and those using three-dimensional solid element, or the difference

Table 1 Stress concentration factors obtained using thin shell, thick shell, and solid elements

\begin{tabular}{|c|c|c|c|}
\hline Poisson's ratio, $v$ & Thin shell (2-D) & Thich shell & Solid (3-D) \\
\hline \multirow{2}{*}{0} & 1.39 & 1.62 & 1.56 \\
\cline { 2 - 4 } & $(1.0)$ & $(1.17)$ & $(1.12)$ \\
\hline \multirow{2}{*}{0.5} & 1.51 & 1.77 & 1.74 \\
\cline { 2 - 4 } & $(1.0)$ & $(1.17)$ & $(1.15)$ \\
\hline \multirow{2}{*}{$1 / 3^{* 1}$} & $1.8^{* 2}$ & 2.1 & - \\
\cline { 2 - 4 } & $(1.0)$ & $(1.16)$ & \multicolumn{3}{|c|}{${ }^{* 1}$ Infinite plate by E. Reissner ${ }^{(8)}$} \\
\hline
\end{tabular}


between stress concentration factors obtained using plane stress elements or plane strain elements and those using three-dimensional solid elements.

\section{Proposal for the Limits of Application of Two-Dimensional Elasticity} Solutions

\subsection{Tension}

The difference between results obtained using two- and three-dimensional analyses is strongly affected by the value of Poisson's ratio, especially in tension. The stress fields inside a plate do not vary in the thickness direction and satisfy a plane stress condition, because there is no Poisson's effect when $v=0$. Thus the two-dimensional elasticity solution can be applied even in an infinitely thick plate. When $v=0.3$, the value of the dimensionless notch depth, $t / \rho$, hardly affects the difference between stress concentration factors obtained using two-dimensional and three-dimensional analyses. Further, the stress concentration factors obtained using a three-dimensional analysis at the notch root on the surface are smaller than the stress concentration factors obtained using a two-dimensional analysis by $10 \%$ when the ratio of $B / \rho=2.1$ (see Fig. 7(a)). In the case when $v=0.5$, the plane strain condition is realized inside even thinner plate compared to when $v=0.3$ (see $t / \rho=1.0$ and 2.0 of $B / \rho=1.0$ in Fig. 6(b)) and the gradient of $\sigma_{\max }$ in the thickness direction is larger when $v=0.5$, because Poisson's effect is maximized for that case. And we found that the difference between stress distributions due to varying notch depth, $t / \rho$, is not negligible around the center of the plate thickness. However, the difference in stress concentration factors between two-dimensional and three-dimensional analyses does not exceed $10 \%$ irrespective of the notch depth when $B / \rho \leq 0.7$.

Based on the above findings, the recommended limits of application of two-dimensional elasticity solutions are shown in Table 2, with an admissible error of up to $10 \%$.

\subsection{Out-of-plane bending}

The difference in stress concentration factors between two-dimensional and three-dimensional analyses increases as the thickness, $B / \rho$, increases and the notch depth, $t / \rho$, decreases; the difference is also affected by Poisson's ratio. However, it should be emphasized that the plate thickness, $B / \rho$, affects the difference in stress concentration factors the most. The difference of stress concentration factors between two-dimensional and three-dimensional analyses in the present study does not exceed $10 \%$ in cases of $B / \rho \leq 0.5$.

Based on the above findings, the recommended limits of application of two-dimensional elasticity solutions are shown in Table 3, with an admissible error of up to $10 \%$.

Table 2 Limits of application of two-dimensional elastic solutions in tension

\begin{tabular}{|c|c|}
\hline Poisson's ratio, $v$ & Maximum value of $B / \rho$ \\
\hline 0 & $\infty$ \\
\hline 0.3 & 2.1 \\
\hline 0.5 & 0.7 \\
\hline \multicolumn{2}{|c|}{ (Admissible error $=10 \%$ ) }
\end{tabular}


Table 3 Limits of application of two-dimensional elastic solutions in bending

\begin{tabular}{|c|c|}
\hline Notch depth, $t / \rho$ & Maximum value of $B / \rho$ \\
\hline$\geqq 0.4$ & 0.5 \\
\hline \multicolumn{2}{|c|}{ (Admissible error $=10 \%$ ) }
\end{tabular}

\section{Conclusion}

The effect of the dimensionless quantities $B / \rho$ and $t / \rho$ and $v$ on the three-dimensional stress fields near a notch root is clarified by our finite element results. In this study, plates having a U-shaped hole of the same root radius are analyzed with varying plate thicknesses and notch depths. The two loading scenarios studied are tension and out-of-plane bending. The limits of application of two-dimensional elasticity solutions are obtained by comparing the results of the three-dimensional analyses with those of the two-dimensional analyses. The conclusions are as follows:

1. The limits of application of two-dimensional elasticity solutions in tension can be determined by considering the dimensionless plate thickness (plate thickness, $B$, divided by the notch-root radius, $\rho$ ). The value of the ratio $B / \rho$ varies with the value of Poisson's ratio. The stress is largest around the center of the plate thickness and when high values of Poisson's ratio are used. Accordingly, attention to the actual value of Poisson's ratio and the location in the plate where stress is being studied are both necessary when applying two-dimensional elasticity solutions.

2. In out-of-plane bending, the value of $B / \rho$ is the primary variable affecting the three-dimensional stress distribution. Thus, the limits of application of two-dimensional elasticity solutions are determined by the plate thickness, $B / \rho$, at least for cases when $t / \rho \geq 0.4$, which is the lower bound for the analyses conducted in the present study.

\section{References}

(1) Nishida, M., Stress Concentration Factors (in Japanese), (1967), Morikita Publishing Ltd.

(2) Kawamoto, T., Applied Theory of Elasticity (in Japanese), (1968), pp. 77-82, Kyoritsu Shuppan Ltd.

(3) Miyamoto, H., Three-Dimensional Theory of Elasticity (in Japanese), (1967), pp. 80-97, Shokabou Publishing Ltd.

(4) Wang, C.T. (translated to Japanese by Inose, Y.), Applied Theory of Elasticity, (1971), pp. 230-236, Morikita Publishing Ltd.

(5) Hirano, F., Research on Stress Concentration Factors for Two-dimensional Elastic Bodies (Report No. 2) (in Japanese), Transactions of the Japan Society of Mechanical Engineers, vol. 16, No. 55 (1950), pp. 52-58.

(6) Hirano, F., Research on Stress Concentration Factors for Two-dimensional Elastic Bodies (Report No. 3), Transactions of the Japan Society of Mechanical Engineers, vol. 17, No. 61 (1951), pp. 12-17.

(7) Kawai, T. and Endoh, T., Analytical Solution on the 3D Stress Concentration Problem of a Plate with a Circular Hole under Uniform Tension at Infinity, Proceedings of International Conference on Computational Mechanics, (1986), v37-42.

(8) Reissner, E., The Effect of Transverse Shear Deformation of Elastic Plates, Journal of Applied Mechanics, vol. 12, No. 2(1945), A69-77.

(9) Nisitani, H., A Parameter for Stress Field in a Notch Corresponding to Stress Intensity Factor in a Crack, Transactions of the Japan Society of Mechanical Engineers, Series A, vol. 49, No. 
447(1983), pp. 1353-1359.

(10) Washizu, K., On the Bending of Isotropic Plates, Transactions of the Japan Society of Mechanical Engineers, vol. 18, No. 68 (1952), p. 47.

(11) Timoshenko, S. and Woinowsky-Krieger, S. (translated to Japanese by Hasegawa, M.), Theory of Plates and Shells (second edition), (1973), pp. 30-41 and 73-81, Brain Tosho Shuppan Ltd.

(12) p. 78 of reference (11). 